\title{
EEG Signal with Feature Extraction using SVM and ICA Classifiers
}

\author{
Chunchu Rambabu \\ Research Scholor \\ Rayalaseema University, Kurnool, Andhra \\ Pradesh, India
}

\author{
B Rama Murthy, Ph.D \\ Faculty of Instrumentation, \\ S.K. University, Anantapur, AndhraPradesh, India
}

\begin{abstract}
Identifying artifacts in EEG data produced by the neurons in brain is an important task in EEG signal processingresearch. Theseartifacts are corrected before further analyzing. In this work, fast fixed point algorithm for Independent Component Analysis (ICA) is used for removing artifacts in EEG signals and principal component analysis (PCA) tool is used for reducing high dimensional data and spatial redundancy. Support vector machine (SVM) tool is used for pattern recognition of EEG signals and the extracted parameters are used to impart cognitive interpretation ability towards autonomous system design.
\end{abstract}

Keywords:EEG signals, Fast ICA, PCA, SVM and Hardware Architecture.

\section{INTRODUCTION}

Electroencephalograms (EEGs) are recordings of the electricalpotentials developed by the brain. Analysis of EEG activity hasbeen achieved principally in clinical settings to identify pathologiesand epilepsies. An interpretationof the EEG is used to visual inspection by a neurophysiologist. EEG technology used many electrodes on thehuman skull, such signals givesinformation indirectly about physiological functions, which are related tothe brain, these signals are verynumerous. The EEG integrated technical devices with embedded intelligence andit allows for Brain-ComputerInterfaces (BCI) to analysis EEG design. BCI is composed of signalcollection and processing, pattern identification and control systems.EEG classification has many number of features, it comes from the fact that are,

(i) EEG signals are non-stationary, thus, features must be computedin a timevarying manner, and

(ii) Number of EEG channelsis large.

For the classification process, a multilayer perceptron (MLP) neural network is trained with the back propagationalgorithm.

\subsection{EEG Signals Measurement}

The EEG signals measurement is crucial for clinical diagnoses and medical research. The capacitive electrodes are using for non-contact measurement to solving the EEG signal problem that does not require conductive gel and skin allergies, which cannot develop during long-term measurement, because capacitive electrodes are frequently used in clinical medicine. It has various methods for performing non-contact biopotential measurements by using capacitive electrodes. It developed a capacitive sensor for ECG and EEG monitoring and a tiny capacitive sensor for conducting various biopotential measurements. Additional voltage buffer is required to effectively convey physiological signals to the monitoring devices and increases the cost and complexity of sensing systems. The capacitive sensors require further packaging, which may limit their applicability. The available conductive fabric are conducting biopotential measurements to popularize the capacitive technique, because EEG signals are low amplitude (approximately 10 to $100 \mu \mathrm{V}$ ), designing EEG monitoring systems and using conventional electrodes. It uses EEG measurements to verify the feasibility of using conductive fabric for capacitive measurements. The EEG signals are collected and preprocessed using special filters and features are extracted using many methods is shown in Figure 1. The sensor nodes transmittingthe EEG states, receives output via constellation mapper as shown in Figure 2. The receiver reproduces the states using feature extraction, PCA and identifiesthe cognitive states as shown in Figure 3.

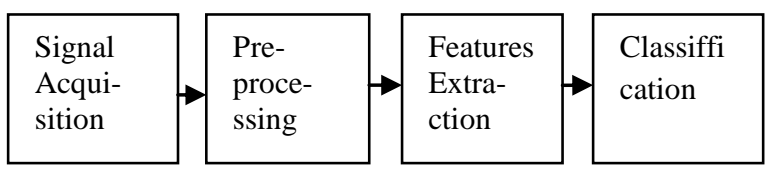

Fig 1: The block diagram of the EEG work process

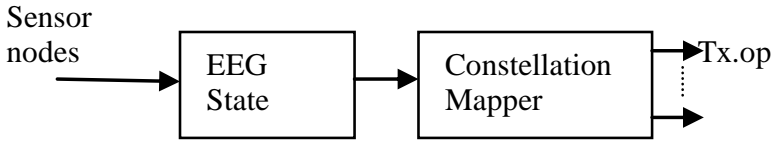

Fig 2: Sensor nodes transmission with EEG states

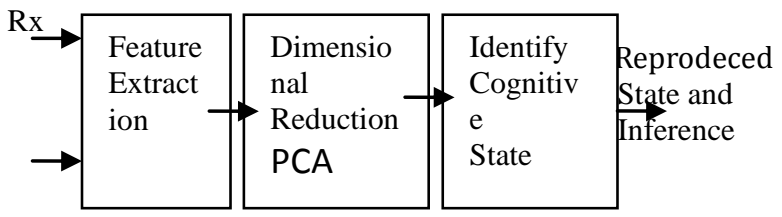

Fig 3: Sample Cognitive states

EEG uses MRI imaging of the brain while performing a cognitive task. It allows detecting the location and magnitude of brain activity involved in the various types of cognitive functions. It also accepts to view and record the changes in the brain activity during the time to perform the task. The analysis of continuous EEG signals or brain waves is complex, due to the large amount of information received from every electrode. Different waves are categorized by the frequency of their emanations, although none of the waves is ever emitted alone. The EEG is recorded between electrodes placed in standard positions on the scalp and has typical amplitude of 2100 microvolts and a frequency spectrum from 0.1 to $60 \mathrm{~Hz}$. EEG activity in particular frequency bands is correlated with cognitive states. Brain-Computer interfaces use EEG signals which can be controlled by the user. These types of EEG signals fall into two main classes; 

evoked by a specific sensory stimulus, such as a flashing light, and

(ii) Spontaneous EEG signals which consist of EEG components that occur without stimulus, such as the alpha rhythm or the mu rhythm.

The ability of subjects to produce strong spontaneous EEG rhythms such as the alpha rhythm or the mu rhythm can be enhanced by the use of biofeedback or operant conditioning, at this process the user is given an indication as to how well he/she is controlling a device. Evoked Potentials (EPs) require a specific external stimulus and originate in sensory cortex areas. A typical evoked potential is the Visual Evoked Potential (VEP), in response to a strobe light for example, the EEG over the visual cortex will vary at the same frequency as the stimulating light. Subjects can be trained to control the strength of their steady state VEP with the use of biofeedback. EEG control signal is controllable frequency which is very easy to detect that means the subsequent signal processing and pattern recognition tasks are very simple.

\section{PREVIOUS WORK}

E. Tamil [1] proposed EEG brain wave feature extraction using short time Fourier transform that externally attaching several electrodes on the human skull.Lee, et al., [2] proposed a low-cost electroencephalograph for task classification, where the EEG potentials recorded at 10-20 EEG electrode positions over the scalp with a cap and integrated electrodes. Molina [3] proposed signal preprocessing which is necessary to maximize the SNR since, many noise sources encountered with the EEG signal. Akrami [4] presented EEG signal is time domain signal and the signal energy distribution is scattered. Behnam, et al., [5] presented to analyze the whole signal, the window is translated in time and FT is reapplied to each one. AbdulhamitSubasi, et al., [6] presented a versatile signal processing and analysis framework for EEG. Cao, et al., [7] proposed PCA, independent component analysis (ICA) and linear discriminant analysis (LDA) methods for feature extraction. Subasi [8] proposed EEG signal classification using wavelet feature extraction and a mixture of expert model. Ubeyli, et al., [9] proposed analysis of EEG signals by combining eigenvector methods and multiclass SVMs.Wang, et al., [10] presented feature extraction and dimensionality reduction algorithms and applications in vowel recognition.

Widodo, et al., [11] proposed feature extraction transforms the existing features into a lower dimensional space which is useful for feature reduction to avoid the redundancy due to high-dimensional data.Carlos, et al., [12] presented a large number of methods for EEG feature extraction demands a good choice for EEG features for every task.Gomez, et al., [13] proposed information theoric feature selection for functional data classification. It excluded from the current feature subset that increases the mutual information when it is discarded.Guerrero, et al., [14] proposed new feature extraction approach for epileptic EEG signal detection using time-frequency distributions. Ocak[15] proposed optimal classification of epileptic seizures in EEG using wavelet analysis and genetic algorithm that can be applied to extract the wavelet coefficients of discrete signals. Marcin et al., [16] presented a new method of feature extraction from EEG signal for brain computer interface design. MiHyeSong, et al., [17] proposed an algorithm for arrhythmia classification, which is associated with the reduction of feature dimensions by LDA and a SVM based classifier. V.V. Shete, et al., [18] proposed detection of K-Complex in sleep EEG signal using SVM. The K-complex is a transient EEG waveform that contributes to the assessment of sleep stages. Sarah, et al., [19] proposed classification of EEG signals using different feature extraction techniques for mental-task BCI.Mohammad et al., [20] presented an automated computer platform for the purpose of classifying EEGsignals associated with left and right hand movements using a hybrid system that uses advanced feature extraction techniques and machine learning algorithms.Kavita, et al., [21] proposed the processing and analysis of EEG within a framework which is carried out with DWT for decomposition of the signal into its frequency subbands.

\section{FEATURE EXTRACTION}

The EEG signal is time domain signal and thesignal energy distribution is scattered. In order to extract the features, theEEG signal is analyzed to give a description of the signalenergy as a function of time and frequency. The features extracted in frequency domain can recognize the mental tasks based on EEG signals. The analysis method is the Fast Fourier Transform(FFT) applies the discrete FFT to the signal and find out its spectrum.EEG signal is non-stationary that means its spectrumchanges with time. Such a signal can be approximated aspiecewise stationary, a sequence of independent stationary signal segments. Although the field of spectral analysis hasbeen dominated by use of the Fourier transform, which do not adequately represent non-stationary signals, the filter process through feature extraction with PCA and SVM classifiers are used as shown in Figure 4.

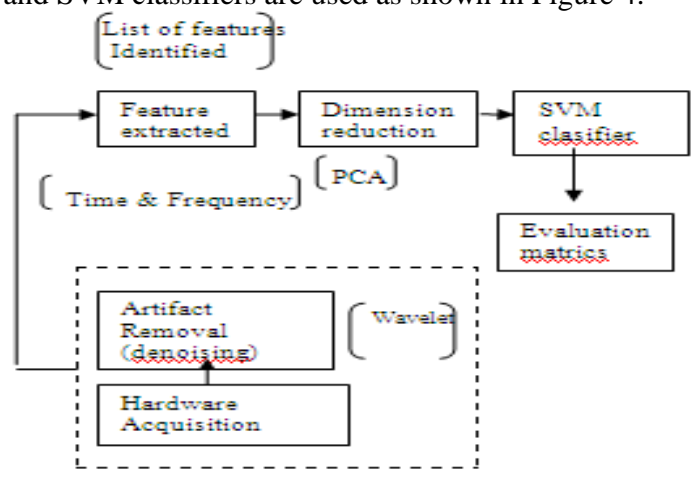

Fig 4: Filter process through feature extraction with classifiers

\subsection{Error Detection using Checksum Analysis}

The data, structure to detect error uses the checksum bits along with the modulation and noise filtering process to extract the cognitive states using demodulation is shown in Figure 5 . 

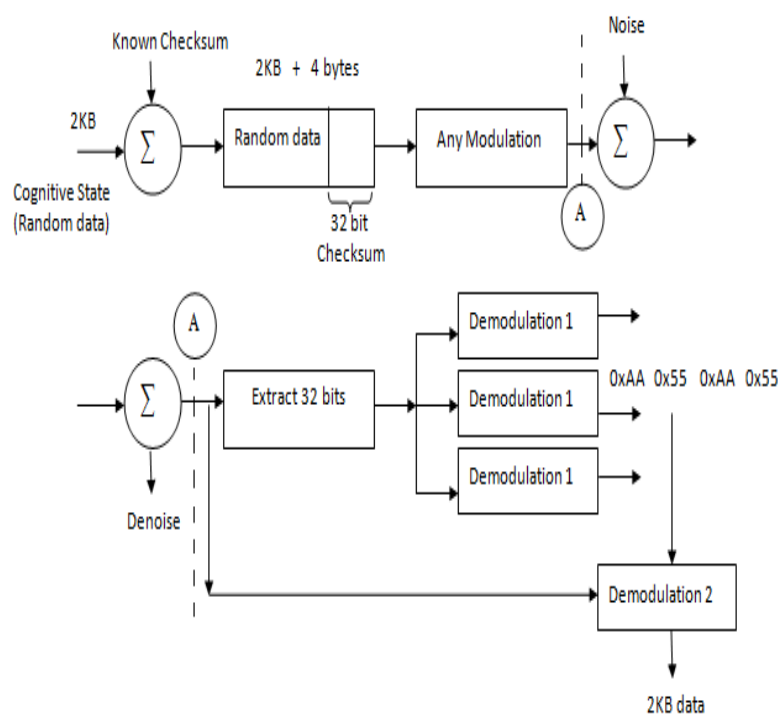

Fig 5: Random data analyze the checksum bits modulation/demodulation process

\section{PRINCIPALCOMPONENT ANALYSIS}

Principal component analysis is used for dimensional data reduction method and to identify underlying variables that are uncorrelated with each other. Intuitively, this is desirable because the underlying variables that account for a set of measured variables should correspond to physically different processes, which, in turn, should have outputs that are uncorrelated with each other.(PCA) is a mathematical procedure that uses an orthogonal transformation to convert a set of observations of possibly correlated variables into a set of values of linearly uncorrelated variables called principal components. The number of principal components is less than or equal to the number of original variables. PCA is the simplest of the true eigenvector-based multivariate analysis. Often, its operation can be thought of as revealing the internal structure of the data in a way that best explains the variance in the data. If a multivariate dataset is visualized as a set of coordinates in a high-dimensional data space (1 axis per variable), PCA can supply the user with a lower-dimensional picture, a "shadow" of this object when viewed from its (in some sense; see below) most informative viewpoint. This is done by using only the first few principal components so that the dimensionality of the transformed data is reduced.

\subsection{PCA Algorithm}

Before applying PCA, must do data preprocessing, which is given a set of ' $m$ ' unlabeled examples that should do the following

(i) Mean normalization: Replace each xji with $x j-\mu j$,

In other words, determine the mean of each feature set, and then for each feature subtract the mean from the value, so we re-scale the mean to be 0

(ii) Feature scaling (depending on data)

If features have very different scales then scale them so they all have a comparable range of values

$$
\text { e.g. } x j i \text { is set to }(x j-\mu j) / s j
$$

Where, sj is some measure of the range, so could be

(a.) Biggest - smallest

(b.) Standard deviation (more commonly)

(iii) With preprocessing done, PCA finds the lower dimensional sub-space which minimizes the sum of the square.

In summary, for 2D->1D follow as shown in the below figure,

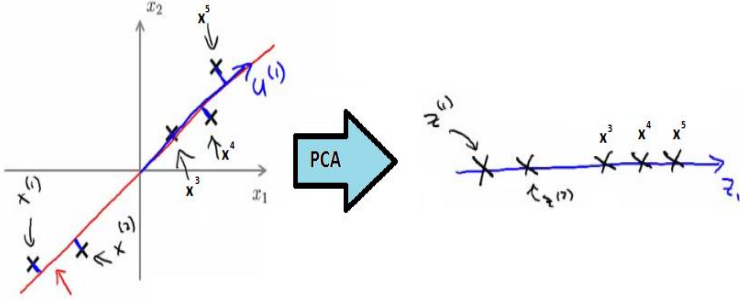

It needs to compute two things;

(i) Compute the ' $u$ ' vectors

(ii) Need to compute the ' $z$ ' vectors, where it has lower dimensionality feature vectors

A mathematical derivation for the ' $u$ ' vectors is very complicated, but once derivation has done, the procedure to find each ' $u$ ' vector is not that hard.

\subsection{Algorithm description}

This algorithm describes the following steps

(a) Reducing data from $n$-dimensional to $\mathrm{k}$ dimensional and compute the covariance matrix

$$
\sum=\left(\frac{1}{m}\right) \sum_{i=1}^{n}\left(\mathrm{x}^{\mathrm{i}}\right)\left(x^{i}\right)^{\mathrm{T}}
$$

This is commonly denoted as $\Sigma$ (greek upper case sigma) NOT summation symbol $(\Sigma)=$ sigma. This is an $[\mathrm{n} \times \mathrm{n}]$ matrix that remember than $\mathrm{x}^{\mathrm{i}}$ is a $[\mathrm{n} \times 1]$ matrix

(b) Compute eigenvectors of matrix $\Sigma$

(c) $[\mathrm{U}, \mathrm{S}, \mathrm{V}]=\operatorname{svd}($ sigma $)$

Where, svd = singular value

decomposition, and

More numerically stable than eig,

Where, eig provides eigenvector

(d) U,S and V are matrices

where, $\mathrm{U}$ matrix is an $[\mathrm{n} \times \mathrm{n}]$ matrix, turns out the columns of $\mathrm{U}$ are the $\mathrm{u}$ vectors, so to reduce a system from $\mathrm{n}$-dimensions to $\mathrm{k}$-dimensions to take the first $k$ vectorsfromU(firstk columns)

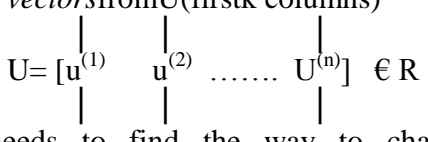

It needs to find the way to change ' $\mathrm{x}$ ' (which is $\mathrm{n}$ dimensional) to $\mathrm{z}$ (which is $\mathrm{k}$ dimensional)

(e) It reduces the dimensionality

(f) Take first ' $k$ ' columns of the ' $u$ ' matrix and stack in columns, where $\mathrm{n} \times \mathrm{k}$ matrix - call this $\mathrm{U}_{\text {reduce }}$

(g) Calculate ' $\mathrm{z}$ ' as follows, $\mathrm{z}=\left(\mathrm{U}_{\text {reduce }}\right)^{T} * \mathrm{x}$ so, $[\mathrm{k} \mathrm{x} \mathrm{n}] *[\mathrm{n} \times 1]$

Generates a matrix which isk $* 1$.

\section{METHODOLODY OF COGNITIVE}

STATES (CS) WITH BITS FEATURES

The Boolean variable is given as 1 or 0 , and different features

\begin{tabular}{|c|c|c|c|c|c|c|}
\hline $1 / 0$ & $\begin{array}{l}\text { Feature } \\
1\end{array}$ & $\begin{array}{l}\text { Feature } \\
2\end{array}$ & $\ldots$ & $\ldots$ & $\ldots$ & $\begin{array}{l}\text { Feature } \\
\mathrm{N}\end{array}$ \\
\hline Bool & & & \multicolumn{4}{|c|}{$\mathrm{N}$ features } \\
\hline
\end{tabular}
are given in Figure 6.

Fig 6: Different features fused in the Data Structure 
Figure 7 shows that all different cognitive states (CS) transmit with 32 bit features, modulations scheme and shows the outputas same CS.

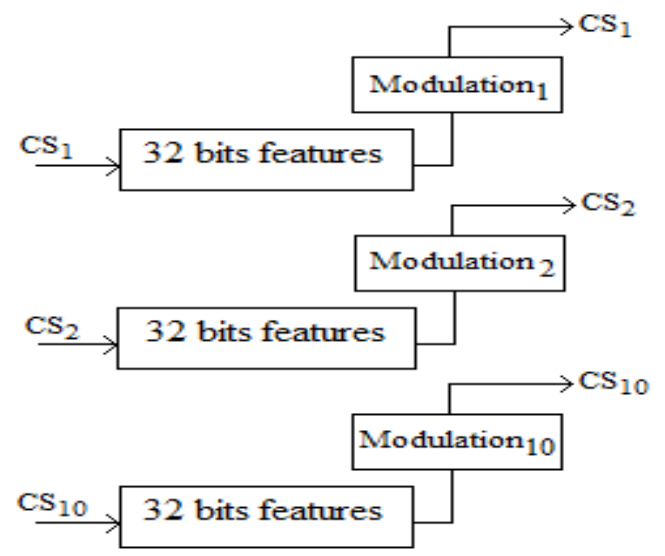

Fig 7: Cognitive states and modulation scheme Every 32-bit data has a header and transmit to modulation and generate output as shown in Figure 8.

Header $+\mathrm{CS}_{1}$ Data

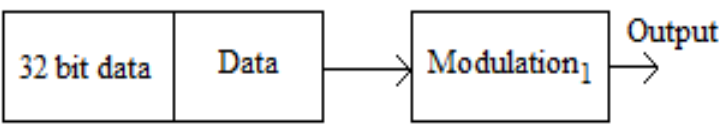

Fig 8: Header and data structure

$\begin{array}{llcc}\text { 0xAA } & \text { 0x55 } & \text { 0xAA } & \text { 0x55 } \\ 10101010 & 01010101 & 10101010 & 01010101\end{array}$

\section{DESCRIPTION OF TEST INPUTS}

The description of the test inputs for cognitive states is shown in Table 1.

Table 1. Cognitive states and recorded EEG signal waves

\begin{tabular}{|c|c|}
\hline Cognitive state & St Recorded EEG W/F \\
\hline 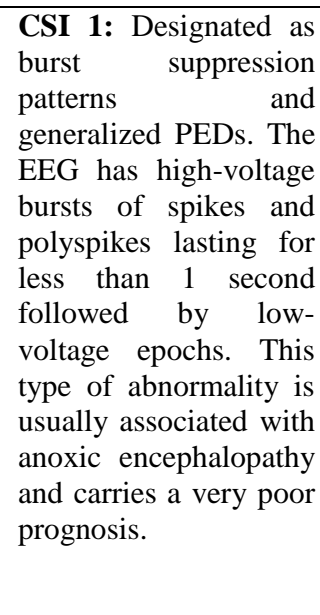 & 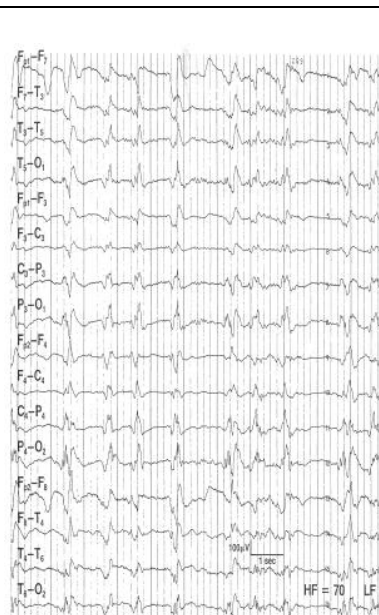 \\
\hline
\end{tabular}

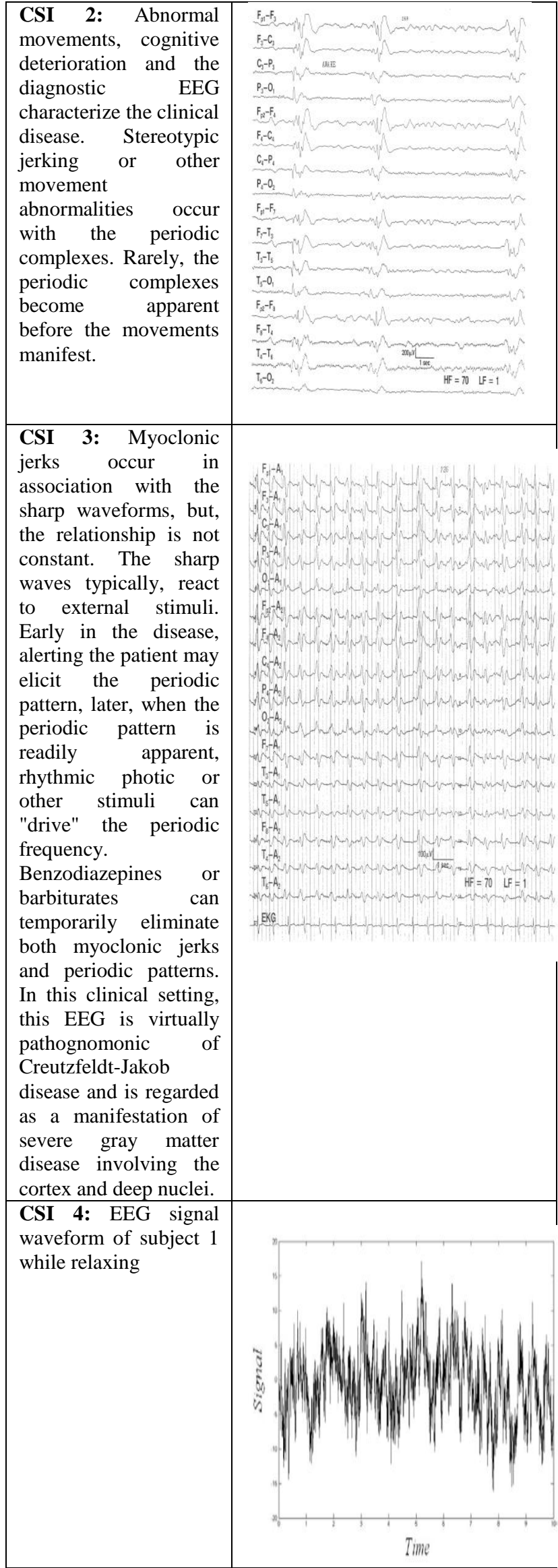




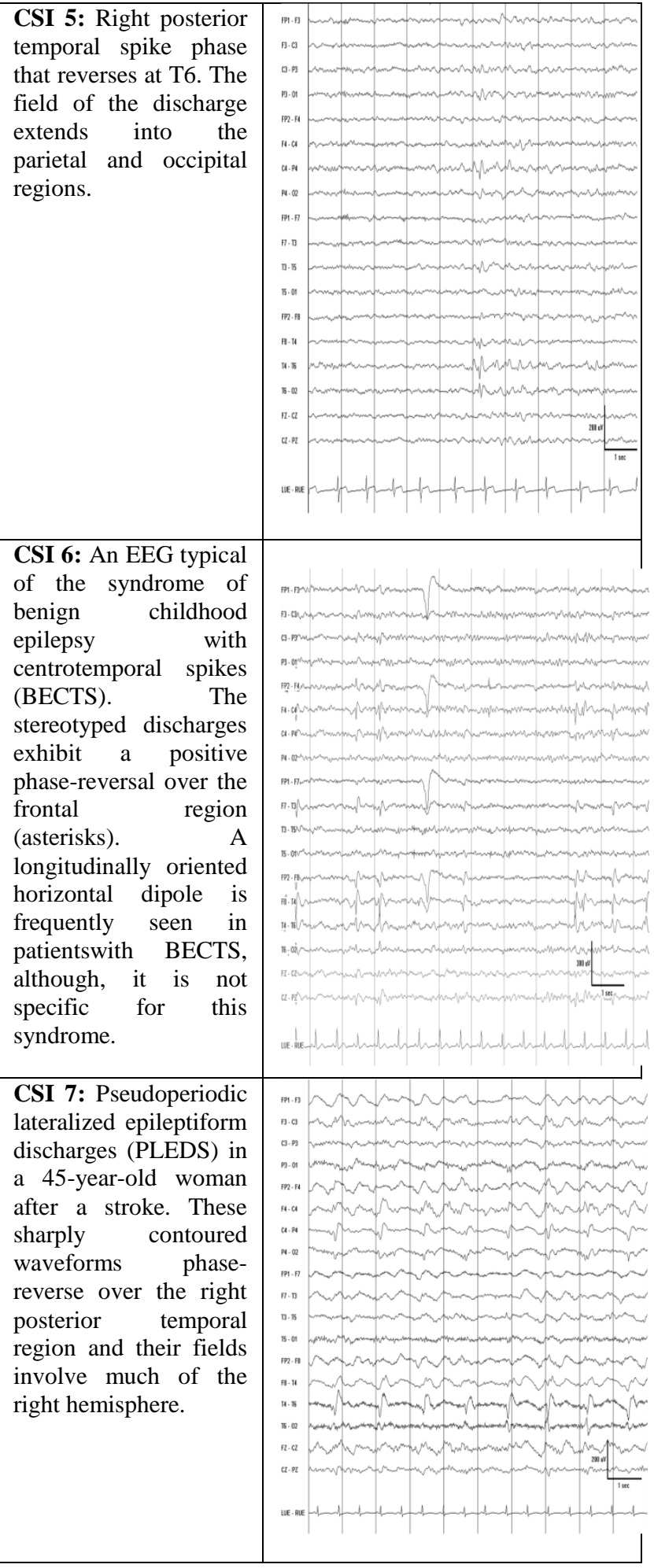

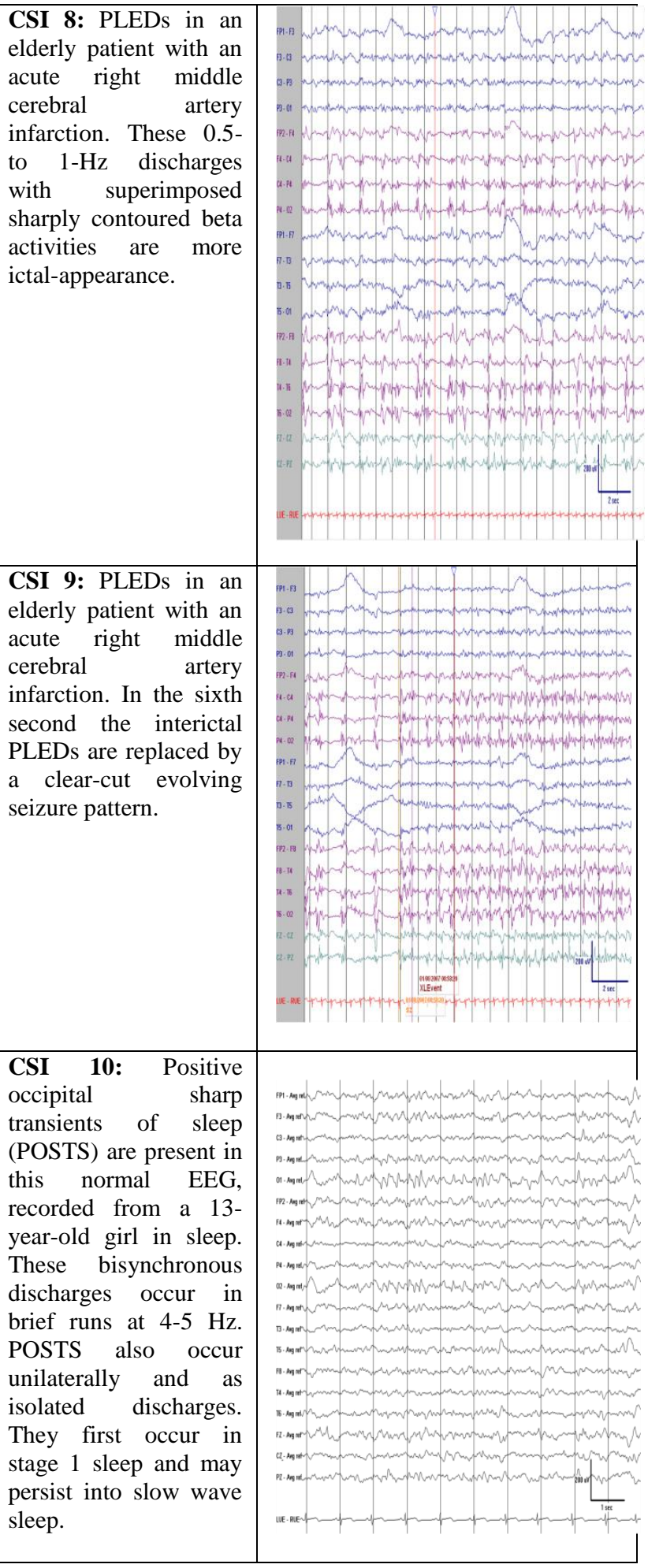




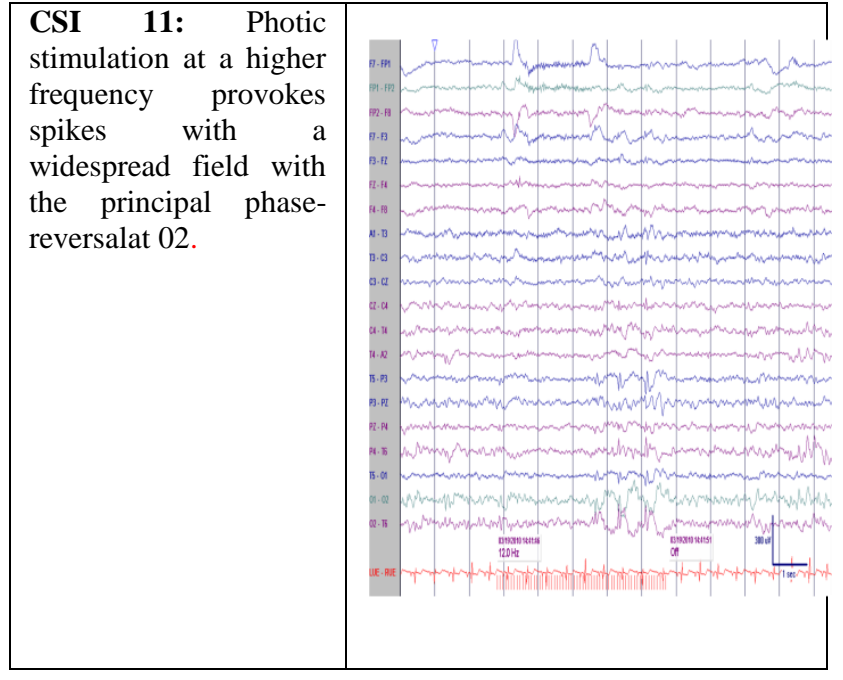

\section{RESULTS AND DISCUSSION}

In this work, cognitive states are simulated at different states with hardware modulation and demodulation and matched states are shown from Figure 9 to Figure 12.

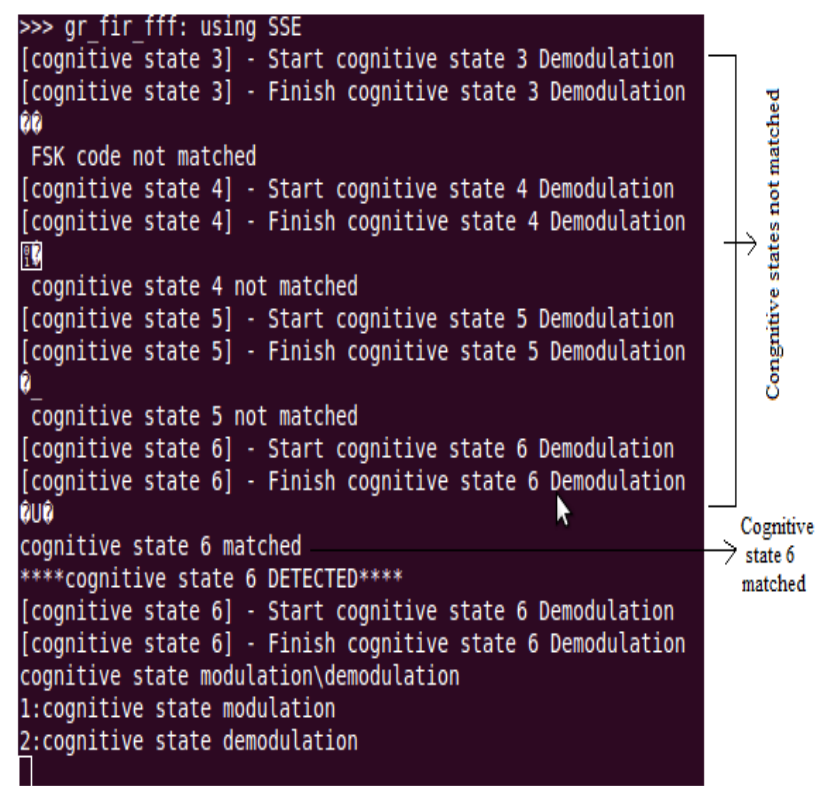

Fig 9: Cognitive state 6 matched and detected

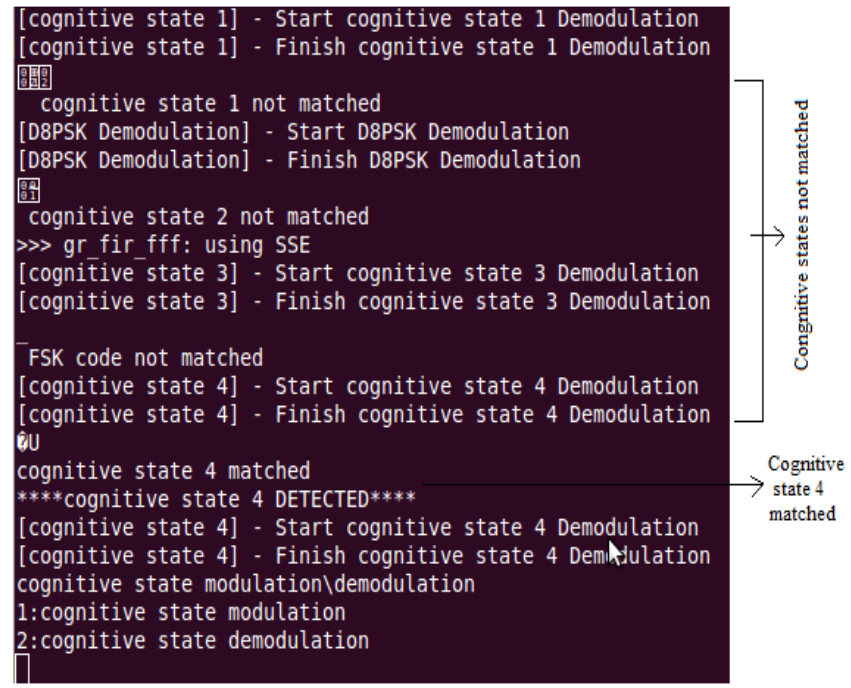

Fig 10: Cognitive state 4 matched and detected

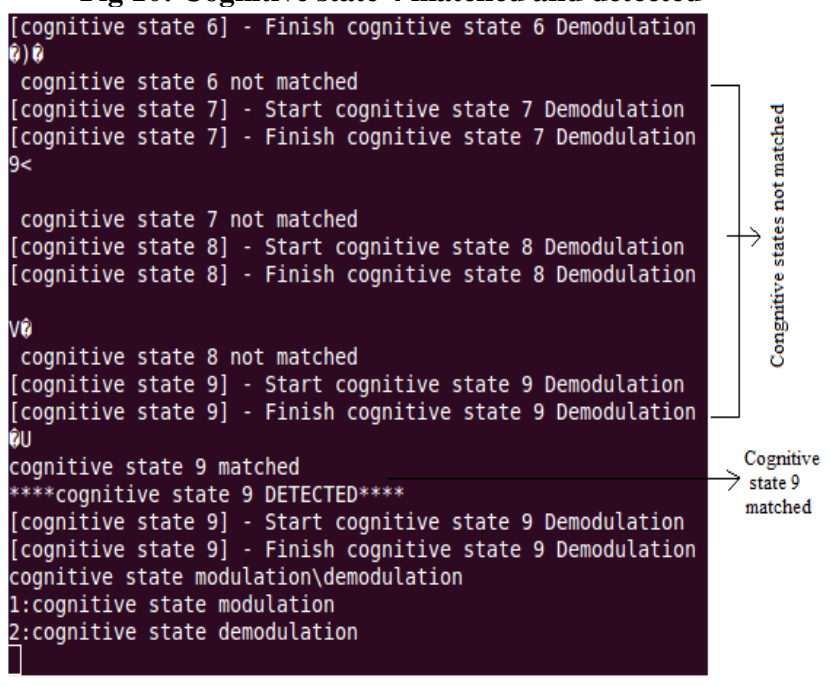

Fig 11: Cognitive state 9 matched and detected

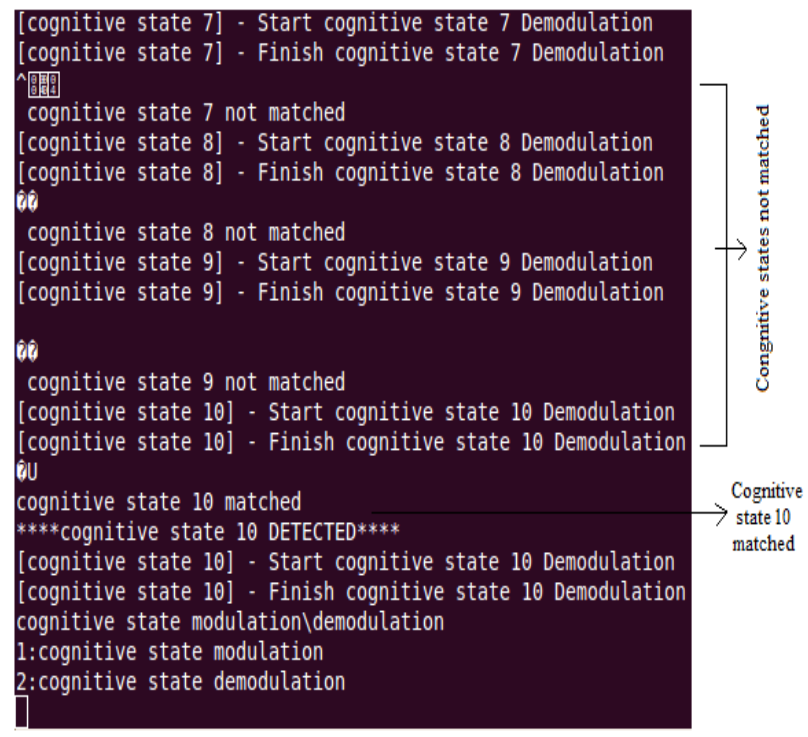

Fig 12: Cognitive state 11 matched and detected 


\section{CONCLUSION}

In this study, it summarizes the overview of artifacts and their removal in EEG signals, where artifacts are the combination of EMG and EOC signals that influence on EEG signals. The EEG signals are collected and pre-processed using special filters then features areextracted. For classification of signals, employ Multi Layer Perceptron (MLP) trained with back propagation algorithm. The datasets were inputted into fast fixed point algorithms and SVMs that has to be analyzed for a better knowledgeable extraction and more accurate decision rules. In this research work, EEG signal is analyzed to provide a description of signal as a function of time and frequency. The features are extracted in frequency domain and analyzed using FFT to find the spectrum of the signal. Since the EEG signal is non stationary its spectrum changes with time, so it is approximated as piecewise stationary and processed for feature extraction with PCA and SVM. Using SVM the patterns of EEG signals are recognized and the parameters are implemented in the SVM hardware. PCA recognize first few principal components and reducing the high dimensional data and spatial redundancy. Thus, the EEG signals with artifacts are finally corrected and removed by employing ICA.

\section{REFERENCES}

[1] E. Tamil,"Electroencephalogram (EEG) Brain Wave FeatureExtraction Using Short Time Fourier Transform", Faculty of ComputerScience and Information Technology, University of Malaya,2007.

[2] J. Lee, D. Tan, "Using a Low-Cost Electroencephalograph for TaskClassification in HCI Research", UIST'06, Montreux, Switzerland, October 15-18, 2006.

[3] G. Molina, "Joint Time-Frequency-Space Classification of EEG ina Brain-Computer Interface Application", EURASIP Journal on AppliedSignal Processing, Vol. 7, pp. 713-729, 2003.

[4] A. Akrami, "EEG-Based Mental Task Classification: Linear andNonlinear classification of Movement Imagery", in proceedings of theIEEE Engineering in Medicine and Biology $27^{\text {th }}$ AnnualConference Shanghai, China, September 1-4, 2005.

[5] H. BehnamA, A. SheikhaniB, M. MohammadiC, M. NoroozianD, P.Golabie, "Analyses of EEG background activity in Autism disorder withfast Fourier transform and short time Fourier transform", InternationalConference on Intelligent and Advanced Systems, 2007.

[6] AbdulhamitSubasi, M. Ismail Gursoy, "EEG signal classification using PCA, ICA, LDA and support vector machines", Expert Systems with Applications, Vol.37, pp. 8659-8666, 2010.

[7] Cao, L. J., Chua, K. S., Chong, W. K., Lee, H. P., \&Gu, Q. M., "A comparison ofPCA, KPCA and ICA for dimensionality reduction in support vector machine", Neurocomputing, 55, pp. 321-336, 2003.

[8] Subasi, A., "EEG signal classification using wavelet feature extraction and amixture of expert model", Expert Systems with Applications, 32, pp. 1084-1093, 2007.

[9] Ubeyli, E. D., "Analysis of EEG signals by combining eigenvector methods andmulticlass support vector machines", Computers in Biology and Medicine, 38,pp. $14-22,2008$
[10] Wang, X., Paliwal, K. K., "Feature extraction and dimensionality reductionalgorithms and their applications in vowel recognition", Pattern Recognition, 36,pp. 2429-2439, 2003

[11] Widodo. A., Yang. B., “Application of nonlinear feature extraction andsupport vector machines for fault diagnosis of induction motors", Expert Systemswith Applications, 33, pp. 241-250, 2007.

[12] Carlos Guerrero-Mosquera, Michel Verleysen and Angel Navia Vazquez, "EEG feature selection using mutualinformation and support vectormachine: A comparative analysis", $32^{\text {nd }}$ Annual International Conference of the IEEE EMBSBuenos Aires, Argentina, August $31^{\text {st }}$ - September $4^{\text {th }}, 2010$.

[13] Gomez V. Vanessa, Verleysen Michel and Jerome Fleury, "Informationtheoric feature selection for functional data classification",Neurocomputing,Vol.72, pp. 3580-3589, 2009.

[14] Guerrero-Mosquera C., MalandaTrigueros A., Iriarte Franco J. andNavia Vazquez Angel, "New feature extraction approach for epilepticEEG signal detection using time-frequency distributions",Med BiolEngComputer, Vol.48, pp. 321-330, 2009.

[15] OcakHasan, "Optimal classification of epileptic seizures in EEG usingwavelet analysis and genetic algorithm",Signal processing, Vol.88, pp. 1858-1867, 2008.

[16] MarcinKoOdziej, AndrzejMajkowski,Remigiusz J. Rak, "A new method of feature extraction from EEG signal for braincomputerinterface design", Przegl D Elektrotechniczny, ISSN 0033-2097, R. 86 NR 9/2010.

[17] MiHye Song, Jeon Lee, Sung Pil Cho, KyoungJoung Lee, and Sun Kook Yoo, "Support Vector Machine Based Arrhythmia ClassificationUsing Reduced Features", International Journal of Control, Automation and Systems, Vol.3, No.4, pp. 571-579, December 2005.

[18] V.V.Shete, SachinElgandelwar, Sapna Sonar, AshwiniCharantimath, Dr.V.D.Mytri, "Detection of KComplex in Sleep EEG Signal using Support Vector Machine", International Journal of Scientific \& Engineering Research, Vol.3, Issue 6, No.1, ISSN 2229. 5518, June-2012.

[19] Sarah M. Hosni, Mahmoud E. Gadallah, Sayed F. Bahgat, Mohamed S. AbdelWahab, "Classification of EEG Signals Using DifferentFeature Extraction Techniques for Mental-Task BCI", IEEE Transactions, 2007.

[20] Mohammad H. Alomari, AyaSamaha, and KhaledAlKamha, "Automated Classification of $\mathrm{L} / \mathrm{R}$ Hand Movement EEG Signals using Advanced Feature Extraction and Machine Learning", International Journal of Advanced Computer Science and Applications(IJACSA), Vol.4, No.6, 2013.

[21] KavitaMahajan, M. R. Vargantwar, Sangita M. Rajput, "Classification of EEG using PCA, ICA and Neural Network", International Journal of Engineering and Advanced Technology (IJEAT), Volume-1, Issue-1, October 2011. 